(C)2007 IEEE. Personal use of this material is permitted. However, permission to reprint/republish this material for advertising or promotional purposes or for creating new collective works for resale or redistribution to servers or lists, or to reuse any copyrighted component of this work in other works must be obtained from the IEEE 


\title{
Quantifying Failure for Risk Based Decision making in Digital Business Ecosystem Interactions
}

\author{
Omar Khadeer Hussain, Elizabeth Chang, Farookh Khadeer Hussain and Tharam S. Dillon \\ Digital Ecosystems and Business Intelligence Institute, Curtin University of Technology, Perth, Australia \\ omar.hussain@postgrad.curtin.edu.au, \{elizabeth.chang, farookh.hussain, tharam.dillon\}@cbs.curtin.edu.au
}

\begin{abstract}
Due to technological advancement of the internet, conducting e-commerce transactions have become a part of our daily lives. In a financial interaction to be carried over the digital business ecosystem domain, it is rational for an agent instigating the interaction to analyse beforehand the possible risk in interacting with any other agent. Doing so would give the instigating agent an idea of direction in which its interaction might head and also help it to make an informed decision of its future course of action with that particular agent. For risk analysis, the instigating agent has to determine beforehand the probability of failure and the possible consequences of failure in interacting with an agent. In this paper, we propose such a methodology by which the instigating agent quantifies the probability of failure beforehand in interacting with an agent according to the demand of its future interaction with it.
\end{abstract}

\section{Introduction}

The advent of the internet has provided its users with numerous mechanisms for conducting or facilitating e-commerce interactions [1]. The terms 'trusting agent' and 'trusted agent' define the two agents participating in an interaction. The former refers to the instigator of the interaction while the latter refers to the agent accepting the request. The significance of the trusting agent to analyse the possible risk before initiating an interaction with a probable trusted agent is substantial. The trusting agent, by analysing the possible risk beforehand, could gain an idea of whether it will achieve its desired outcomes from the interaction or not. Based on this, it can safeguard its resources. Risk plays a central role in deciding whether to proceed with a transaction or not. It can broadly be defined as an attribute of decision making that reflects the variance of its possible outcomes. The Australian and New Zealand Standard on Risk Management, AS/NZS 4360:2004, states that Risk Identification is the heart of Risk Management [2].
Therefore, by identifying and analysing the possible risk beforehand in interacting with a probable trusted agent, the trusting agent can make an informed decision of its future course of action with it. Risk analysis is important in the study of behaviour in ecommerce interactions because there is a whole body of literature based in rational economics that argues that the decision to buy is based on the risk-adjusted cost-benefit analysis [3]. Thus, it commands a central role in any discussion of e-commerce that is related to a transaction. The need to distinguish between the likelihood and magnitude of risk is important as they represent different concepts.

Digital Business Ecosystems is a new concept that is emerging worldwide as an innovative approach to support the adoption and development of information and communication technologies. Digital ecosystems transcend the traditional, rigorously defined, collaborative environments from centralised or distributed or hybrid models into an open, flexible, domain clustered and demand-driven interactive environment. A digital ecosystem is a new-networked architecture and collaborative environment that addresses the weakness of client-server, peer-to-peer, grid and web services. It is a self-organising digital infrastructure aimed at creating a digital environment for networked organisations that supports the cooperation, knowledge sharing, development of open and adaptive technologies and the evolutionary business models [4-6]. It can also be defined as a system which is loosely coupled, agent-based collaborative environment where every specie is proactive and responsive and acts for its own benefit or profit. A demand driven business ecosystem interaction implies, that the trusting agent wants to achieve certain desired outcomes in its interaction, and in order to attain its goals and outcomes it should select a trusted agent who can fulfil those. In doing that, it is possible the trusting agent has to decide whether to interact or not with a probable trusted agent, or choose on a trusted agent to interact with among a set of probable trusted agents. The trusting agent can ease its decision making process by analysing the possible risk in 
interacting with each of the probable trusted agents according to its demand. The possible risk in an interaction is a combination of the probability of failure in achieving the outcome; and the possible consequences of failure.

In this paper, as a step towards analysing the possible risk in an interaction beforehand, we propose a methodology by which the trusting agent can determine the probability of failure in interacting with a probable trusted agent to achieve its demand. We will propose and explain the methodology in the next sections.

\section{Related Work}

To quantify and express semantically the probability of failure of an interaction, we proposed and defined the term 'FailureLevel' and 'Failure scale' in Hussain et al. [8]. The failure scale, as shown in Figure 1, represents the different possible levels of failure that could be possible in an interaction. 'FailureLevel' (FL) quantifies and expresses semantically the probable level of failure in the interaction on the failure scale. To determine beforehand the probability of failure in interacting with a probable trusted agent, the trusting agent should determine its FailureLevel by ascertaining its incapability to complete the interaction according to the expectations of its future interaction. In other words, the FailureLevel of an interaction is the extent to which the trusting agent determines that it might not achieve its desired outcomes in interacting with a probable trusted agent.

\begin{tabular}{|c|c|c|c|}
\hline \begin{tabular}{|l|} 
Semantics of \\
Failure Level \\
\end{tabular} & $\begin{array}{l}\text { Probability of } \\
\text { Failure }\end{array}$ & FailureLevels & Star Rating \\
\hline Unknown & & -1 & Not Displayed \\
\hline Total Failure & $\begin{array}{c}91-100 \% \text { Probability of } \\
\text { Failure }\end{array}$ & 0 & Not Displayed \\
\hline Extremely High & $\begin{array}{c}71-90 \% \text { Probability of } \\
\text { Failure }\end{array}$ & 1 & From 20 to 2 \\
\hline Largely High & $\begin{array}{l}51-70 \% \text { Probability of } \\
\text { Failure }\end{array}$ & 2 & From \\
\hline High & $\begin{array}{c}26-50 \% \text { Probability of } \\
\text { Failure }\end{array}$ & 3 & From \\
\hline Significantly Low & $\begin{array}{l}\text { 11- } 25 \% \text { Probability of } \\
\text { Failure }\end{array}$ & 4 & From WWW to thW \\
\hline Extremely Low & $\begin{array}{l}0-10 \% \text { Probability of } \\
\text { Failure }\end{array}$ & 5 & From WhW \\
\hline
\end{tabular}

Figure 1 showing the Failure scale

The expectations or the desired outcomes that the trusting agent wants in its interaction with a probable trusted agent can be at a higher level termed as the 'context' of the interaction. In other terms context represents the high level nature of the trusting agent's interaction with the probable trusted agent [7]. It can be decomposed into several detailed aspects known as the criteria. Criteria is defined as the demand or the set of factors which show specifically what the trusting agent wants in its interaction with the trusted agent in the particular context. Further in this paper we label the desired outcomes or the expectations of the trusting agent as the 'criteria' in the interaction. By considering the expectations of its future interaction, the trusting agent will accurately determine the probability of failure in interacting with a probable trusted agent according to its criteria.

The possible interaction of the trusting agent with the probable trusted agent is in the future state of time. Hence, to determine the FailureLevel of the probable trusted agent in that state of time, we propose that the trusting agent should analyse the probability of failure in interacting with it in two stages. They are:

1. Pre-Interaction start time phase

2. Post-Interaction start time phase

Pre-Interaction start time phase refers to the period of time before the trusting agent starts its interaction with the probable trusted agent, whereas PostInteraction start time phase is that period of time, after the trusting agent starts and interacts with the probable trusted agent. For risk analysis, the trusting agent has to determine the FailureLevel in interacting with a probable trusted agent in this period of time, that is in the post-interaction start time phase. However as this time phase is in the future state of time, the trusting agent can only determine it by using some prediction methods. So to achieve this we propose that the trusting agent should first ascertain the FailureLevel of the probable trusted agent according to the specific context and criteria as that of its future interaction, in the pre-interaction start time phase. Based on those achieved levels, the trusting agent can determine its FailureLevel in the post-interaction start time phase. The determined FailureLevel in that time phase depicts the probability of failure in interacting with the probable trusted agent, during the time of its interaction with it.

In the next sections we will propose the methodologies by which the trusting agent can determine the FailureLevel of a probable trusted agent, in the two different time phases according to the context and criteria of its future interaction with it.

\section{Time Based FailureLevel Analysis}

We define risk in the context of a decentralized financial e-commerce interaction as the likelihood that the probable trusted agent will not act as expected by the trusting agent resulting in the failure of the interaction and loss of resources involved in it [8]. This 'likelihood' varies throughout the transaction 
depending on the behaviour of the probable trusted agent and, therefore, it is dynamic. As mentioned in the literature too, risk is dynamic and varies according to time. It is not possible for an agent to have the same impression of a trusted agent throughout, which it had at a particular time. Hence, the trusting agent should take into account this dynamic nature of risk while doing risk analysis in its interaction with a probable trusted agent.

In order to incorporate and consider this dynamic nature, we propose that the trusting agent should determine the FailureLevel in interacting with a probable trusted agent in regular intervals of time. By doing so it ascertains the correct FailureLevel of the probable trusted agent in a time interval, according to its incapability to complete the criterions of its future interaction in that particular interval of time, thus considering its dynamic nature while doing risk analysis. We will define some terms by which the total time can be divided into different separate intervals.

We quantify the level of failure on the failure scale in interacting with a probable trusted agent in a given context and at a given time ' $t$ ' which can be either at the current, past or future time with the metric 'FailureLevel'. But for better understanding, we represent the FailureLevel of a probable trusted agent according to the time phase in which they are determined and they correspond to. For example, if the FailureLevel for a probable trusted agent is determined in the pre-interaction start time phase, then we represent it by the metric 'PFL' which stands for 'Previous FailureLevel'. Similarly, if the FailureLevel for the probable trusted agent is determined in the postinteraction start time phase we represent it by ' $\mathrm{FFL}$ ' which stands for 'Future FailureLevel'.

We define the total boundary of time which the trusting agent takes into consideration to determine the FailureLevel (previous or future) of a probable trusted peer as the time space.

As mentioned earlier, risk varies according to time and if the time space is of a long duration, then the FailureLevel of the probable trusted agent might not be the same throughout. Hence, we propose that the time space is divided into different non-overlapping parts and the trusting agent determines the FailureLevel of the probable trusted agent in each of those parts, according to its incapability to complete the criterions to reflect it correctly while doing risk analysis. These different non-overlapping parts are called time slots.

The time at which the trusting agent or any other agent giving recommendation deals with the probable trusted agent in the time space is called a time spot.

The trusting agent should first decide about the total time space over which it is going to analyse the FailureLevel of a probable trusted agent. Within the time space, the trusting agent should determine the duration of each time slot. Once it knows the duration of each time slot, it can determine the number of time slots in the given time space and then subsequently analyse the FailureLevel of the probable trusted agent in each time slot, be it either in past or future.

For explanation sake, let us suppose that a trusting agent wants to interact with a probable trusted agent for a period of 7 days from 24/01/2007 until $30 / 01 / 2007$. This is the post-interaction start time phase. Before initiating the interaction, the trusting agent wants to determine the probability of failure of the interaction as a first step towards risk analysis. To achieve that, the trusting agent wants to assess the reputation of the probable trusted agent, to determine its FailureLevel according to the criteria of its future interaction with it, from a period of 21 days prior to starting an interaction with it, which is from $03 / 01 / 2007$ to $23 / 01 / 2007$. This is the pre-interaction start time phase. Hence, the total period of time which the trusting agent takes into consideration to determine the FailureLevel (PFL and FFL) of the probable trusted agent is of 28 days. This time space is a combination of pre- and post-interaction start time phase. Further, the trusting agent wants to analyse the FailureLevel of the probable trusted agent on a weekly basis. So, the total time space is of 28 days and each time slot is of 7 days. The number of time slots in this time space will be 4 as shown in Figure 2.

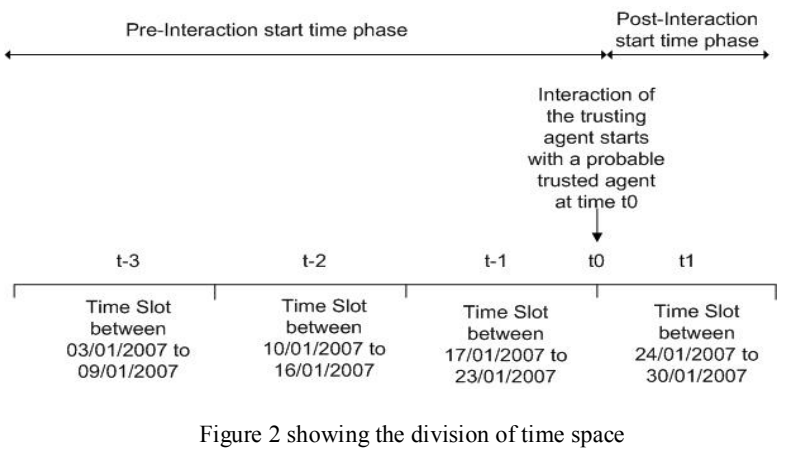

Hence the trusting agent by determining the FailureLevel of the probable trusted agent in different time slots within the time space of its interaction will consider its accurate dynamic level of failure, according to its incapability to complete the criterions in those time slots and reflecting it while doing risk analysis. In the next section we will define the methodology by which the trusting agent can determine the FailureLevel of a probable trusted agent in each time slot according to the specific time phase it is in. 


\section{Determining the FailureLevel in the Pre-Interaction time phase}

As mentioned earlier, pre-interaction time phase is the period of time before the trusting agent interacts with a probable trusted agent. The trusting agent can determine the FailureLevel of a probable trusted agent in each of the pre-interaction start time slots of its interaction either by:

a) Considering its past interaction history with the probable trusted agent, if it was in that time slot and in the criteria as that of its future interaction with it; or

b) Soliciting recommendations from other agents and determining the in-capability of the probable trusted agent to complete the interaction according to the criteria of its future interaction with it.

If the trusting agent has a previous interaction history with the probable trusted agent in a preinteraction time slot and according to the criteria of its future interaction, then it can analyse that interaction to determine the FailureLevel of the probable trusted agent in that time slot. On the other hand if the trusting agent does not have any previous interaction history with the probable trusted agent in a pre-interaction time slot, or in the criteria of its interest, then it can determine its FailureLevel in that time slot, by soliciting recommendations from other agents and then determining the trusted agent's in-capability, on the failure scale to complete the interaction according to its expectations.

Some factors to be considered by the trusting agent while assimilating the recommendations and determining the FailureLevel of the probable trusted agent in the pre-interaction time phase are:

1. The trustworthiness of the recommendations: It is possible that the recommending agent is communicating un-trustworthy recommendations or recommendations which the trusting agent finds to vary considerably. The trusting agent should omit such recommendations before it assimilates them.

2. The time of the recommendations: This is the time at which the recommending agent interacted with the probable trusted agent in question. Before assimilating the recommendations, the trusting agent should ensure that the time of the recommendation corresponds to the time slot in which it wants to determine the FailureLevel of the probable trusted agent by assimilating it.

3. The criteria of the recommending agent's interaction with the probable trusted agent: An important point to be understood is that even if the context of two interactions is the same, their criteria might differ considerably. Hence, the trusting agent should consider only those recommendations whose criterions are of interest to it in its future interaction with the probable trusted agent before assimilating them.

We have defined a methodology in Hussain et al. [9] by which the trusting agent assimilates the recommendations and determines the FailureLevel (PFL) of the probable trusted agent on the failure scale, in each time slot of the pre-interaction time start phase. Due to space limitation, we will just give a brief explanation of the methodology and will not discuss it in detail here.

- By assimilating the recommendations in a preinteraction time slot, the trusting agent determines the FailureLevel of the probable trusted agent in each criterion of its future interaction with it.

- It then combines the FailureLevel of each criterion with the significance of that criterion, to determine a crisp FailureLevel of the probable trusted agent in that time slot. The significance of each criterion shows the degree to which it influences the successful outcome of the interaction, according to the trusting agent.

- The trusting agent while assimilating the recommendations gives more weight to those which are from trustworthy recommending agents as compared to those which are from unknown recommending agents. The recommendations from untrustworthy recommending agents are omitted and not considered.

- Also the trusting agent gives more weight to those recommendations which are in the recent time slot, to the time spot of its interaction with a probable trusted agent, as compared to those which are in the far recent ones, so as to reflect the current status or reputation of that probable trusted agent.

Once the trusting agent determines the FailureLevel (PFL) of the probable trusted agent in each of the pre-interaction time slots, according to the context and criteria of its future interaction with it, then it can determine the FailureLevel (FFL) of the probable trusted agent in the post-interaction start time slots. In the next section, we will propose the methodology of determining the future FailureLevel of a probable trusted agent on the failure scale in the post-interaction start time phase.

\section{Determining the FailureLevel in the Post-Interaction time phase}

Post-Interaction time phase refers to the period of time in which the trusting agent might interact with a probable trusted agent and for risk analysis the trusting agent has to determine the FailureLevel (FFL) of the probable trusted agent in this period of time. To 
achieve that, we propose the trusting agent should utilise the FailureLevel of the probable trusted agent that it had determined according to the specific context and criteria as that of its future interaction in the preinteraction start time slots and then, based on those levels, it should predict its FailureLevel in the postinteraction time slot. Hence, from Figure 2, if the trusting agent has to determine the FailureLevel of a probable trusted agent in time slot $\mathrm{t} 1$ it should consider the FailureLevel (PFL) of that trusted agent, that it had determined from time slots $\mathrm{t}-3$ till $\mathrm{t}-1$ in the preinteraction phase by assimilating the recommendations and then utilise those to determine its FailureLevel at time slot $\mathrm{t} 1$. As the determined FailureLevel (PFL) of a probable trusted agent in the pre-interaction start time phase is strictly according to the criteria of its future interaction, the future FailureLevel (FFL) determined by utilising these levels too is strictly according those criteria.

We propose that the trusting agent while determining the FailureLevel (FFL) of a probable trusted agent in a post-interaction time slot, should determine the probability of occurrence of each level of failure within the domain of $(0,5)$ on the failure scale, rather than determining a crisp FailureLevel as it does in the pre-interaction time slots. This is because the future FailureLevel (FFL) of a probable trusted agent at time $t+1$ is predicted by considering its FailureLevels from its time space till time $t$. This might not give an accurate conclusion as compared to the one obtained in the pre-interaction time slots where the trusting agent determines the trusted agent's FailureLevel by either considering its past interaction history or by assimilating the recommendations. In order address this; we propose that the trusting agent in each post-interaction time slot should determine the magnitude of occurrence of each level of failure on the failure scale, rather than determining a crisp FailureLevel, in interacting with the trusted agent. By doing so the trusting agent would also determine the probability of occurrence of each level of failure on the failure scale in interacting with the probable trusted agent in a post-interaction time slot, according to its criteria.

Our method for determining the future FailureLevel (FFL) for a probable trusted agent at time slot $\mathrm{t}+1$ is by taking its FailureLevels from the beginning of the time space till time $t$ and utilise the Gaussian Distribution to determine the probability of the future FailureLevel (FFL) being any level on the failure scale. As mentioned, the failure scale ranges from $(-1,5)$, with -1 denoting unknown level of failure and levels between 0 and 5 denoting varying degree of failure levels. So the future FailureLevel of a probable trusted agent in a post-interaction time slot is determined in the domain of $(0,5)$ on the failure scale. Within this domain there are six possible levels of failure on the failure scale.

To determine a trusted agent's future FailureLevel at time $t+1$ within each level on the failure scale, let us suppose that the trusting agent has determined the FailureLevel of that trusted agent in each time slot from the beginning of the time space of its interaction till time $t$. Those FailureLevel of the trusted agent from the time space till time $t$ are represented as:

$$
\left\{\mathrm{FL}_{1}, \mathrm{FL}_{2}, \mathrm{FL}_{3} \ldots \mathrm{FL}_{\mathrm{K}}\right\}
$$

where $\mathrm{k}$ is the number of time slots.

The mean FailureLevel $\left(\mu_{\mathrm{FL}}\right)$ is calculated as:

$\mu_{\mathrm{FL}}=\frac{1}{K} \sum_{i=1}^{K} F L i$ .....Equation 1

Accordingly the unbiased sample variance $\left(\sigma^{2}\right)$ is:

$\sigma^{2}=\frac{1}{K-1} \sum_{i=1}^{K}\left(\mathrm{FL}_{\mathrm{i}}-\mu_{\mathrm{FL}}\right)^{2} \quad$.....Equation 2

Since the future FailureLevel FFL $\sim\left(\mu_{\mathrm{FL}}, \sigma^{2}\right)$, then for any random variable FFL, according to Gaussian distribution [10] the probability of it being in a given range $(a, b)$, where $a<b$, and $a, b$ are within the domain of $(0,5)$ on the failure scale can be determined by:

$$
\mathrm{P}(\mathrm{a}<\mathrm{FFL} \leq \mathrm{b})=\frac{1}{\sqrt{2 \prod} \sigma} \int_{\frac{a-\mu}{\sigma}}^{\frac{b-\mu}{\sigma}} \mathrm{e}^{\frac{-t 2}{2}} \mathrm{dt}
$$

Hence in each post-interaction time slot, the probability of FFL of a probable trusted agent is determined for each level within the domain of $(0,5)$ on the failure scale, by utilising its previous failure levels. Further, by representing the FFL of the probable trusted agent in each post-interaction time slot by bus bars of the different possible levels of failure, the trusting agent would get a better indication of the degrees of failure in that time slot and how it might behave in that time slot. The proposed concepts will be understood and its significance can be determined more clearly when we explain it in the next section by taking an example.

\section{Example of determining the FailureLevel in the Post-Interaction phase}

To demonstrate the proposed concept of determining the FailureLevel (FFL) of a probable trusted agent in the post-interaction time phase, let us consider that a trusting agent ' $\mathrm{A}$ ' wants to interact with a trusted agent for a period of one week from $24 / 01 / 2007$ to $30 / 01 / 2007$ in the context of transporting its goods of worth $\$ 20,000$. For simplicity, 
we term the context as ' $\mathrm{C}$ ' and assume the criteria that the trusting agent wants in its interaction are ' $\mathrm{C} 1$ ' and ' $\mathrm{C} 2$ '. There are two logistic companies, agent ' $\mathrm{B}$ ' and agent ' $\mathrm{D}$ ' who can fulfil the trusting agent A's request. These agents are a set of probable trusted agents from which the trusting agent has to decide to interact with. The trusting agent does not have any previous interaction history with these agents in any context and in order to make an informed decision of choosing an agent to interact with, it decides to analyse the possible risk present in interacting with each of them. Risk analysis is a combination of determining the probability of failure and the possible consequences of failure in an interaction.

The trusting agent wants to interact with a probable trusted agent from $24 / 01 / 2007$ to $30 / 01 / 2007$. This is the post-interaction time phase. To determine the FailureLevel (FFL) of the probable trusted agents in this time phase, agent ' $A$ ' decides to analyse the FailureLevel (PFL) of each probable trusted agent according to the context and criteria of its future interaction, for a period of 3 weeks prior to the time spot of its interaction with them, that is from $03 / 01 / 2007$ to $23 / 01 / 2007$. Subsequently, the total time space in which agent ' $A$ ' will be analysing and determining the FailureLevel of the probable trusted agents is from $03 / 01 / 2007$ to $30 / 01 / 2007$. This time space is the combination of pre- and post-interaction time phase. Agent 'A' divides the time space into four different non-overlapping time slots, each of 7 days to determine the FailureLevel of each probable trusted agent in each of them. The time spot of agent A's interaction with any probable trusted agent is 24/01/2007 and, hence, it has to:

- Determine the FailureLevel (PFL) of each probable trusted agent in the pre-interaction start time phase which is from $03 / 01 / 2007$ to $23 / 01 / 2007$ by soliciting for recommendations and then ascertaining their incapability to complete the interaction according to its future criteria, and

- By predicting the future FailureLevel (FFL) of each probable trusted agent in the time space of 24/01/2007 to $30 / 01 / 2007$ to determine the FailureLevel in the post-interaction start time phase.

Let us assume that the trusting agent ' $A$ ' solicits and assimilates the recommendations, to determine the FailureLevel of the probable trusted agents ' $B$ ' and ' $D$ ' on the failure scale according to the criteria ' $\mathrm{C} 1$ ' and ' $\mathrm{C} 2$ ' in the pre-interaction time slots ' $t-3$ ' till ' $t-1$ '. Utilising these FailureLevel the trusting agent should then determine the FailureLevel of the trusted agents in the post-interaction phase.

\subsection{Determining FailureLevel of agent ' $B$ '}

Let us consider the determined FailureLevel of agent ' $\mathrm{B}$ ' on the failure scale in the pre-interaction time slots are:

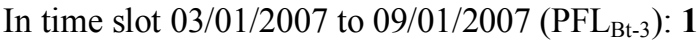

In time slot 10/01/2007 to $16 / 01 / 2007\left(\mathrm{PFL}_{\mathrm{Bt}-2}\right): \mathbf{2}$

In time slot 17/01/2007 to 23/01/2007 (PFL $\left.\mathrm{PLt}_{\mathrm{B}-1}\right): \mathbf{3}$

Determining the mean FailureLevel $\mu_{\mathrm{FL}}=2$

Accordingly, the Sample Variance $\left(\sigma^{2}\right)$ is: $\sigma^{2}=1 ; \sigma=1$

As mentioned earlier, in a post-interaction time slot the trusting agent should determine the probability of occurrence of each level of failure on the failure scale, while determining the FFL in interacting with a probable trusted agent. Using equation 3 to determine the probability of the future FailureLevel (FFL) of agent ' $\mathrm{B}$ ' in each level on the failure scale in time slot t1:

$$
\begin{aligned}
& \mathrm{P}(0<F F L<0.4)=0.032=3.20 \% \\
& \mathrm{P}(0.5<F F L<1.4)=0.2075=20.75 \% \\
& \mathrm{P}(1.5<F F L<2.4)=0.3469=34.69 \% \\
& \mathrm{P}(2.5<F F L<3.4)=0.2277=22.77 \% \\
& \mathrm{P}(3.5<F F L<4.4)=0.0586=5.86 \% \\
& \mathrm{P}(4.5<F F L<5)=0.0049=0.49 \%
\end{aligned}
$$

Representing in Figure 3 the probability of occurrence of each failure level:

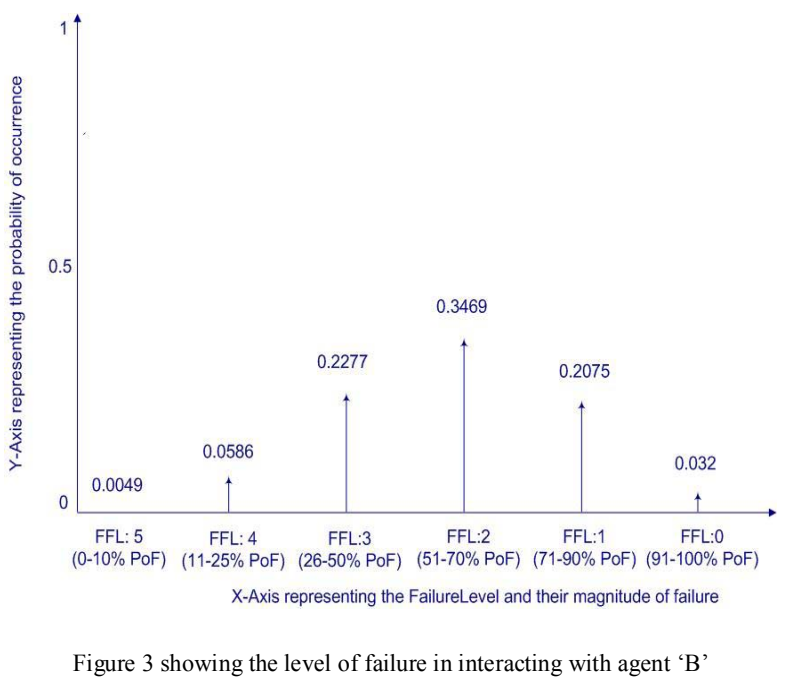

\subsection{Determining FailureLevel of agent ' $D$ '}

Let us consider the determined FailureLevel of agent ' $\mathrm{D}$ ' on the failure scale in the pre-interaction time slots are:

In time slot 03/01/2007 to 09/01/2007 (PFL $\left.{ }_{D t-3}\right): 2$

In time slot 10/01/2007 to 16/01/2007 (PFL $\left.{ }_{D t-2}\right): 2$ 
In time slot 17/01/2007 to 23/01/2007 (PFL $\left.\mathrm{Pt}_{\mathrm{Dt}-1}\right): 0$

Determining the mean FailureLevel $\mu_{\mathrm{FL}}=1.33$

Accordingly, the Sample Variance $\left(\sigma^{2}\right)$ is:

$$
\sigma^{2}=1.33 ; \sigma=1.154
$$

Using equation 3 to determine the probability of the future FailureLevel (FFL) of agent ' $D$ ' in each level of the Failure scale in time slot t 1 :

$$
\begin{aligned}
& \mathrm{P}(0<F F L<0.4)=0.0726=7.26 \% \\
& \mathrm{P}(0.5<F F L<1.4)=0.285=28.50 \% \\
& \mathrm{P}(1.5<F F L<2.4)=0.2655=26.55 \% \\
& \mathrm{P}(2.5<F F L<3.4)=0.1195=11.95 \% \\
& \mathrm{P}(3.5<F F L<4.4)=0.0262=2.62 \% \\
& \mathrm{P}(4.5<F F L<5)=0.0024=0.24 \%
\end{aligned}
$$

Representing the probability of occurrence of each failure level in Figure 4:

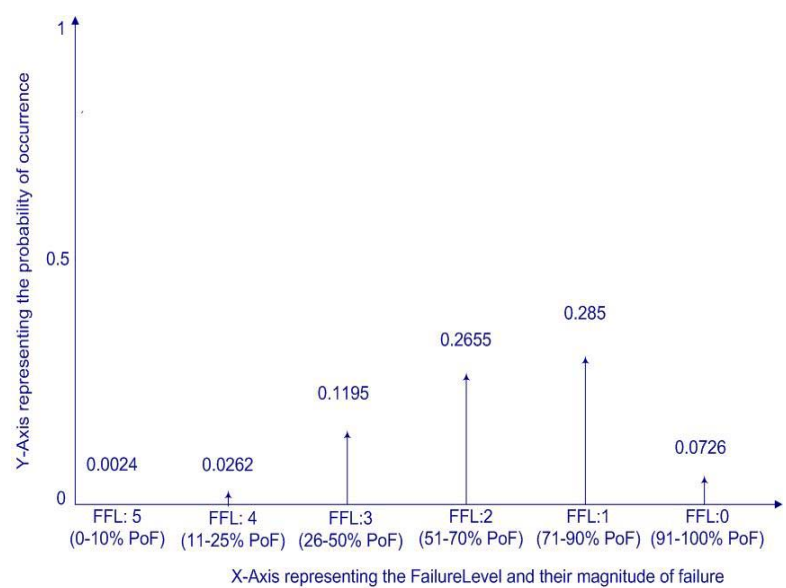

Figure 4 showing the level of failure in interacting with agent ' $D$ '

Figure 3 and 4 show the probability of occurrence of each level of failure on the failure scale in interacting with the probable trusted agents ' $\mathrm{B}$ ' and ' $\mathrm{D}$ ' respectively in time slot $t 1$, according to the criteria of the trusting agent's future interaction with them. The trusting agent ' $A$ ' by analysing the degree of failure of each level, and the probability of occurrence of that level, in interacting with a probable trusted agent can get an idea of how that agent will behave in that time slot and hence the level of failure or its FailureLevel in completing the interaction according to its criteria.

If the trusting agent has more than one time slot in the post-interaction time phase of its interaction, then for risk analysis, it has to determine the FailureLevel (FFL) in interacting with the probable trusted agents in each of the post-interaction start time slots. To achieve that, we propose the trusting agent after determining the probability of occurrence of each level of failure, in interacting with a probable trusted agent in a postinteraction time slot, should take the level with the highest probability of occurrence as the FailureLevel of that probable trusted agent for that particular time slot. Taking our example, if the trusting agent ' $A$ ' has to determine the FailureLevel of the probable trusted agents ' $\mathrm{B}$ ' and ' $\mathrm{D}$ ' in two time slots (t1 and $\mathrm{t} 2$ ) of the post-interaction time phase, then it can take the FailureLevel (FFL) of agent ' $\mathrm{B}$ ' and agent ' $\mathrm{D}$ ' for time slot $\mathrm{t} 1$, as ' 2 ' and ' 1 ' respectively on the failure scale, as these are the individual levels with the highest probability of occurrence in that time slot for these agents. It can then use the methodology defined in section 4 and consider the time slots from t-3 till t 1 while determining the FailureLevel of each probable trusted agent in time slot $\mathrm{t} 2$.

Once the trusting agent determines the FailureLevel of the probable trusted agents in each time slot of its post-interaction phase, it should then determine the possible consequences of failure to its resources, to ascertain the possible risk in interacting with each agent. This is our future work.

\section{Conclusion}

In this paper we emphasised the need for the trusting agent to analyse the possible risk before initiating an interaction with a probable trusted agent, according to its criteria in a digital business ecosystem domain. The possible risk in an interaction is a combination of the probability of failure and the possible consequences of failure in an interaction. We proposed a methodology by which the trusting agent can determine beforehand the probability of failure in interacting with a probable trusted agent according to the criteria of its interaction with it, and also demonstrated the proposed methodology with an example.

\section{References}

[1] H. Chan, R. Lee, T.S. Dillon and E. Chang, 'E-Commerce and its Applications', 1st edition, John Wiley and Sons, Ltd, 2002.

[2] D. F. Cooper, 'The Australian and New Zealand Standard on Risk Management, AS/NZS 4360:2004', Tutorial Notes: Broadleaf Capital International Pty Ltd, Available: $\mathrm{http}: / /$ www.broadleaf.com.au/tutorials/Tut_Standard.pdf, 2004.

[3] S. Greenland, 'Bounding analysis as an inadequately specified methodology', Risk Analysis, vol. 24, no. 5, 2004, pp. 1085-1092.

[4] Technologies for Digital Ecosystems, Available: http://www.digital-ecosystems.org/, Retrieved on 6 October 2006

[5] G.I. Doukidis, N. Mylonopoulos and N. Pouloudi, 'Social and economic transformation in the digital era', Idea Group International, ISBN: 1591402670, 2003.

[6] P.J. Denning, R.M. Metcalfe and M. Robert, 'Beyond calculation: The next fifty years of computing', (edited) Springer ISBN: 0387985883, 1998.

[7] E. Chang, T. Dillon, F. K. Hussain, 'Trust and Reputation for Service-Oriented Environments: Technologies for Building Business Intelligence and Consumer Confidence', 1st edition, John Wiley and Sons Ltd, ISBN: 0-470-01547-0, 2006.

[8] O.K. Hussain, E. Chang, F.K.Hussain and T.S. Dillon, 'A Methodology for Risk Measurements in e-Transactions', Special issue of International Journal of Computer System, Science and Engineering, vol 21, no. 1, 2006, pp. 17-31

[9] O.K. Hussain, E. Chang, F. K. Hussain \& T. S. Dillon, 'Towards Ascertaining Risk [9] O.K. Hussain, E. Chang, F. K. Hussain \& T. S. Dillon, 'Towards Ascertaining Risk
in Digital Business Ecosystem Interactions' IEEE conference on Innovations and Information Technology, Dubai, November 2006, pp. 1-5. [10] N.A. Weiss, 'A Course in Probability', Pearson Education, Inc., USA, ISBN 0-201-
$77471-2,2006$. 